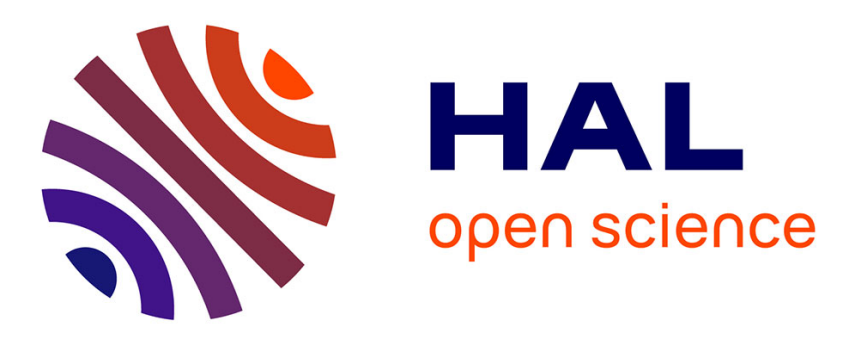

\title{
Contribution to continuum estimation in gamma spectrum by observation of local minima
}

Kélian This, Laurent Le Brusquet, Adrien Frigerio, Sébastien Colas, Pascal Bondon

\section{- To cite this version:}

Kélian This, Laurent Le Brusquet, Adrien Frigerio, Sébastien Colas, Pascal Bondon. Contribution to continuum estimation in gamma spectrum by observation of local minima. 24th International Conference on System Theory, Control and Computing, Oct 2020, Sinaia, Romania. pp.937-942. hal-02985542

HAL Id: hal-02985542

https://hal-centralesupelec.archives-ouvertes.fr/hal-02985542

Submitted on 11 Jan 2021

HAL is a multi-disciplinary open access archive for the deposit and dissemination of scientific research documents, whether they are published or not. The documents may come from teaching and research institutions in France or abroad, or from public or private research centers.
L'archive ouverte pluridisciplinaire HAL, est destinée au dépôt et à la diffusion de documents scientifiques de niveau recherche, publiés ou non, émanant des établissements d'enseignement et de recherche français ou étrangers, des laboratoires publics ou privés. 


\title{
Contribution to continuum estimation in gamma spectrum by observation of local minima
}

\author{
This Kélian ${ }^{1 *}$, Le Brusquet Laurent ${ }^{2 *}$, Frigerio Adrien ${ }^{1}$, Colas Sébastien $^{1}$, Bondon Pascal ${ }^{2}$
}

\begin{abstract}
This paper presents a method to estimate the continuum of a gamma rays spectrum through the observation of local minima. The method is simple, automatable and has a large field of use. Indeed, it is not limited by the peaks width, and consequently is usable as well with GeHP as with scintillators spectra. As the method exploits signal properties, its operation is easily explainable. It involves a limited set of meaningful parameters for which an adjustment is proposed. The potential of this method is demonstrated through simulations as well as real gamma spectrometry measurement.
\end{abstract}

Keywords: gamma spectrometry, continuum estimation, baseline correction method, peak characterization, local minima

\section{INTRODUCTION}

Gamma spectrometry is a common nuclear measurement technic which can be used for the detection of radioactivity, identification of radionuclides, and quantification of radioactive material. Eventhough other methods exist, in practice, the gamma spectrometry often constitutes the only possible and effective technic. As a consequence, gamma spectrometry has become essential in the nuclear field.

One will find in [1] a complete description of gamma rays Physics as well as a number of details on the measurement device. The result of a measurement is a histogram, called spectrum, which spreads detected photons by channels each corresponding to an interval of energy. All spectra have the same structure, that is to say a superposition of a background with peaks specific to some radionuclides, covered by an observation noise. Peaks are mathematically described by a mixture model, usually Gaussian, which contains a great deal of useful information. On the opposite, the background, also called continuum which is rather regular and smooth (at least, in view of the peaks), contains few information.

The purpose of the spectrum analysis is to estimate the mixture parameters from the data. Consequently, continuum is of little interest and the big step of the spectrum analysis will be to isolate the mixture from the background. This operation is known as baseline correction, and the objective of this study is to propose a Baseline Correction Method (BCM) adapted to gamma spectrometry. An ideal method enables the automation of the analysis. Therefore, it shall deal with various peaks shapes and widths, with various radiation detectors technologies, i.e. GeHP (Germanium Hyper Pur, or High Purity

\footnotetext{
${ }^{1}$ Commissariat à l'énergie atomique et aux énergies alternatives (CEA), Valduc, France

${ }^{2}$ Laboratoire des Signaux et Systèmes (L2S), Université Paris-Saclay, CNRS, CentraleSupélec, Gif-sur-Yvette, France
}

GermaniumRadiation) detectors as well as scintillators. The method shall admit a reduced set of parameters independent from the observation.

Spectrum analysis is a recurrent topic in gamma spectrometry, and was a highly topical issue in the 70 s where many propositions were made [2]. This period brought to light two distinct strategies. On one hand, local analysis relying on the prerequisite estimate of the peaks positions [3] [4] [5] [6] [7] [8] which enables to estimate pieces of continuum beneath the peaks. One the other hand, global analysis which aims to estimate the whole continuum [9] [10] [11] [12] [13] [14] [15] [16] [17]. Nowadays, those propositions were naturally ranked by the operating experience, and the local approach based upon the second derivative method [3] is commonly used [18] and officially recommended [19]. This method chiefly draws its success from its simplicity and explainability. However, it remains difficult to automate, because it may fail in the presence of Compton edges or multiplets, i.e. mixtures of close overlapping peaks. On the other hand, a large pan of proposed global methods involve a model for the continuum (splines, Gaussian processes etc), which introduce an improper regularity prior: continuum often contains discontinuities which are difficult to model. Thus, spectrum analysis is still an active research topic.

The central idea of the present study relies on the following empirical observation: local minima rarely appear on peaks. Thus, it would be possible to estimate the continuum from local minima. Several authors [9] [10] [11] have beat around this idea, but the work of Tervo et al. [12] is the most accomplished: it enables to simply estimate the continuum without any prior nor parameters. However, as illustrates the application on two representative spectrum shown in Fig. 1, this estimator only works for thin peaks which quickly limits its use for real applications.

The present paper proposes a new method which covers a much wider range of spectra configurations as shown Fig. 1. The resulting process is in itself simple to describe, but maybe not easy to understand at first glance. The focus is put upon the statistical phenomenon which enables the method to work.

Section II, on one hand, gives a definition of the spectrum. On the other hand, it deducts a number of inherent signal properties on which is built the continuum estimation procedure in section III. Section IV comments the Fig. 1 real spectra application, and section $\mathrm{V}$ concludes this work. 

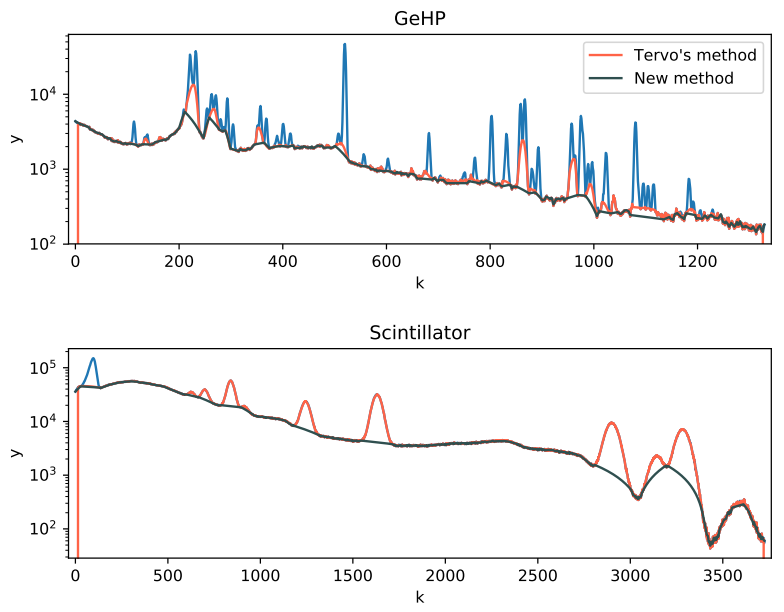

Fig. 1. Confrontation of continuum estimation methods on real spectra. Blue line represents observations. On top is a GeHP spectrum, on the bottom a scintillator spectrum. Both are plotted with a log scale. Red lines represent the reference estimates of Tervo's method. Black lines represent the new method results, applied with parameters $t_{\mathrm{break}}=1.5, w_{\mathrm{opt}}=15$ and $w=10$ for $\mathrm{GeHP}, w=80$ for scintillator.

\section{SPECTRUM SIGNAL PROPERTIES}

This section aims at formalizing the problem and derives some general properties about a gamma spectrum and its components.

\section{A. Basic assumptions}

Let $\boldsymbol{y}$ denote the observed gamma spectrum of $n$ channels such that $\boldsymbol{y}=\left(y_{1}, \ldots, y_{n}\right)$. Let $\boldsymbol{m}=\left(m_{1}, \ldots, m_{n}\right)$ denote the peaks mixture and $\boldsymbol{c}=\left(c_{1}, \ldots, c_{n}\right)$ the continuum. Denoting $\mathcal{P}$ the Poisson's distribution, Physics states [1] $\boldsymbol{y}$ is a sample from a random vector $Y$ such that:

$$
Y_{k} \sim \mathcal{P}\left(m_{k}+c_{k}\right)=\mathcal{P}\left(\mu_{k}\right)
$$

We introduced here $\boldsymbol{\mu}=\boldsymbol{m}+\boldsymbol{c}$, the noiseless signal. Poisson distribution is not practical to handle in literal calculations. Denoting $\mathcal{N}$ the normal distribution, the following approximation is possible [20]:

\section{Hypothesis 1:}

$$
\left\{\begin{aligned}
Y_{k} & \sim \mathcal{N}\left(\mu_{k}, \sigma_{k}^{2}\right) \\
\hat{\sigma}_{k}^{2} & =y_{k}
\end{aligned}\right.
$$

The issue can now be specified: knowing $\boldsymbol{y}$, how to estimate $\boldsymbol{c}$ ? Because $\boldsymbol{m}$ is also unknown, the problem is unsolvable at this state: a constraint is missing. In the paragraphs below, one is looking through the definitions of peaks and continuum for a discrimination criterion that may play the role of the missing constraint.

\section{B. Signal characterization}

Let introduce the differential operator $\Delta x_{k}=x_{k}-x_{k-1}$. The continuum is characterized by its low variations. Thus, continuum variations are majorated:

$$
\exists \beta, \quad \forall k, \quad\left|\Delta c_{k}\right| \leq \beta
$$

A peak has characteristic areas. A top, at the center, has high values and low variations. Two flanks, uprising and downrising on both sides of the top have high variations, especially in view of continuum variations. Two flats at the borders have low values and low variations. Let denote $\mathbb{F}$ the set of all flanks in the spectrum. Then, $\overline{\mathbb{F}}$ contains all tops and flats. The borders of the areas are thereby defined by means of an arbitrary threshold $\alpha$ such that:

$$
\beta \leq \alpha, \quad \forall k \in \mathbb{F}, \quad \alpha \leq\left|\Delta m_{k}\right|
$$

The unfixed threshold $\alpha$ is a necessary scaling variable, and its choice is a matter of convention. Indeed, what could be considered as a peak in a certain context could be considered as a continuum contribution in another. Fig. 2 shows a mono peak signal with a constant continuum. Consequently 0 is the smallest possible value for $\beta$. Choosing $\alpha=50$, resulting $\mathbb{F}$ areas are represented with grey bands.

As a consequence of the previous definitions, one can derive a lower bound for the variations of the signal:

Property 1:

$$
\forall k \in \mathbb{F}, \quad \alpha-\beta \leq\left|\Delta \mu_{k}\right|
$$

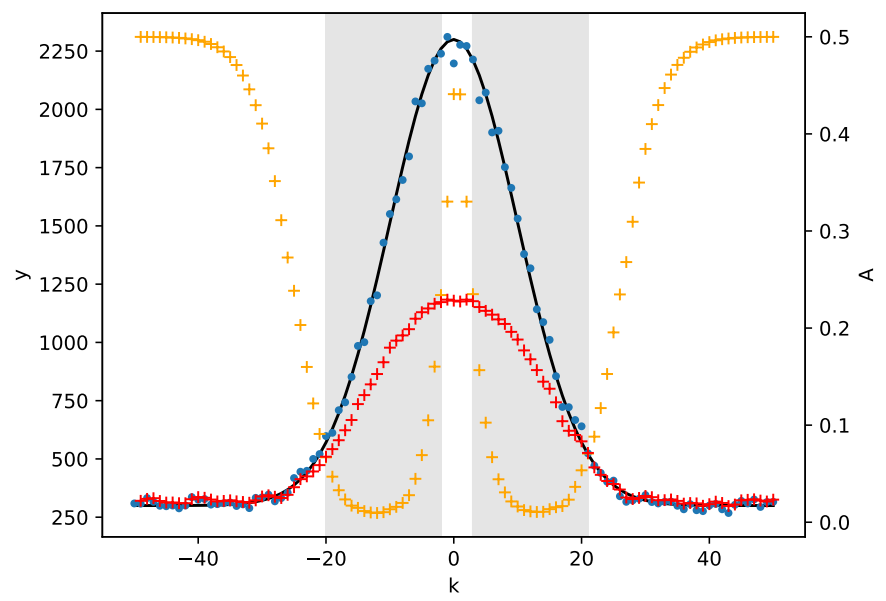

Fig. 2. Identification of peak's areas on a Gaussian example. Dark full line represents $\boldsymbol{m}+\boldsymbol{c}$ signal, blue points represent observations $\boldsymbol{y}$, grey bands indicates $\mathbb{F}$, orange crosses represent $\boldsymbol{A}^{\text {lim }}$, red crosses represent $\boldsymbol{A}$.

\section{Counter variations}

Let denote respectively $\mathbb{F}^{+}$and $\mathbb{F}^{-}$the set of increasing flanks and the set of decreasing flanks:

$$
\left\{\begin{array}{l}
\mathbb{F}^{+}=\left\{k \in \mathbb{F} \mid \alpha \leq \Delta m_{k}\right\} \\
\mathbb{F}^{-}=\left\{k \in \mathbb{F} \mid \Delta m_{k} \leq-\alpha\right\}
\end{array}\right.
$$

$\forall k \in \mathbb{F}$ let $F_{k}$ be the probability to have a counter-variation in $\boldsymbol{y}$ at $k$. More explicitly, $F_{k}$ is the probability for $\boldsymbol{y}$ to decrease where $\boldsymbol{m}$ is increasing, or to increase where $\boldsymbol{m}$ is decreasing:

$$
\begin{aligned}
F_{k} & =P\left(\Delta Y_{k} \leq 0 \mid k \in \mathbb{F}^{+}\right) \\
& =P\left(0 \leq \Delta Y_{k} \mid k \in \mathbb{F}^{-}\right)
\end{aligned}
$$


Notice that $F_{k}$ is almost the repartition function of $\Delta Y_{k} \sim$ $\mathcal{N}\left(\Delta \mu_{k}, y_{k}+y_{k-1}\right)$ evaluated at 0 . Let denote $\Phi($.$) the$ cumulative distribution function (CDF) of the standard normal distribution. Thanks to property 1 and noticing $\Phi$ is an increasing function, one has an upper bound for $F_{k}$ :

Property 2:

$$
\forall k \in \mathbb{F}, \quad F_{k} \leq A_{k}=\Phi\left(\frac{-(\alpha-\beta)}{\sqrt{y_{k}+y_{k-1}}}\right)
$$

On Fig. 2 is plotted $\boldsymbol{A}=\left(A_{1}, \ldots, A_{n}\right)$ for $\alpha=50$ and $\beta=0$. Because this signal is a simulation, one exactly knows the value of $\Delta m_{k}$, which enables to eval the limit admissible values for $A$ as follows:

$$
A_{k}^{\lim }=\Phi\left(\frac{-\Delta m_{k}}{\sqrt{y_{k}+y_{k-1}}}\right)
$$

One notes through $A^{\text {lim }}=\left(A_{1}^{\text {lim }}, \ldots, A_{n}^{\text {lim }}\right)$ that countervariations probabilities are close to zero on high variations areas. This observation is confirmed Fig. 3 where the value of $A_{k}$ quickly decreases.

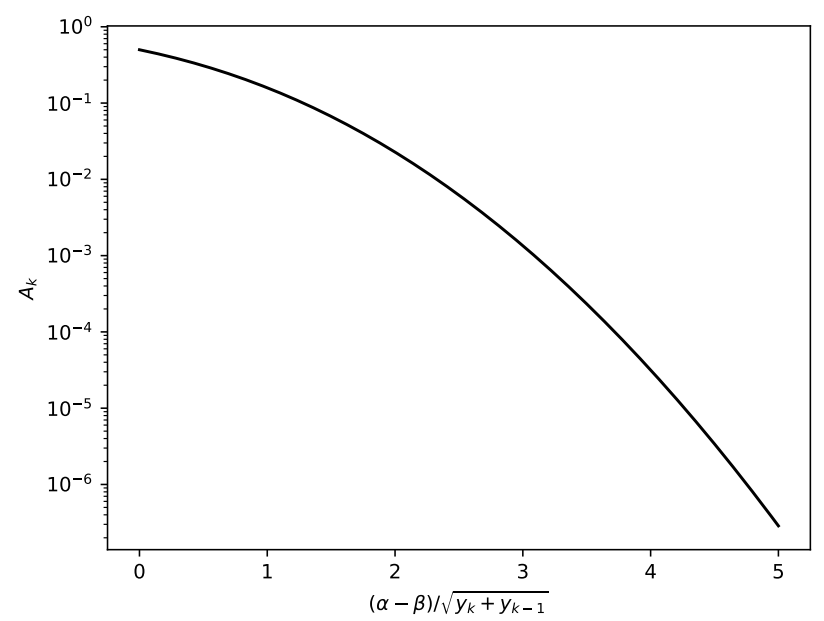

Fig. 3. $A_{k}$ values on a $\log$ scale.

\section{Focus on local minima}

Let introduce $\xi$, the set of indexes of $\boldsymbol{y}$ local minima:

$$
\xi=\left\{k \mid y_{k}<y_{k-1}, y_{k}<y_{k+1}\right\}
$$

1) Local minima bias: As shown in Fig. 4, the local minima set is biased because local minima's expectation is not equal to the signal expectation. Let $\varphi($.$) denote the probability density$ function (PDF) of the standard normal distribution.

Property 3: $\hat{\mu_{\xi_{i}}}$ is an unbiaised estimator of $\mu_{\xi_{i}}$ such that:

$$
\left\{\begin{aligned}
\hat{\mu_{i}} & =y_{\xi_{i}}-\frac{C_{1}}{C_{0}} \sqrt{y_{\xi_{i}}} \\
\operatorname{var}\left(\hat{\mu_{\xi_{i}}}\right) & =\frac{1}{C_{0}}\left(C_{0} y_{\xi_{i}}^{2}+\left(2 C_{1}-C_{0}+C_{2}\right) y_{\xi_{i}}-C_{1} \sqrt{y_{\xi_{i}}}\right)
\end{aligned}\right.
$$
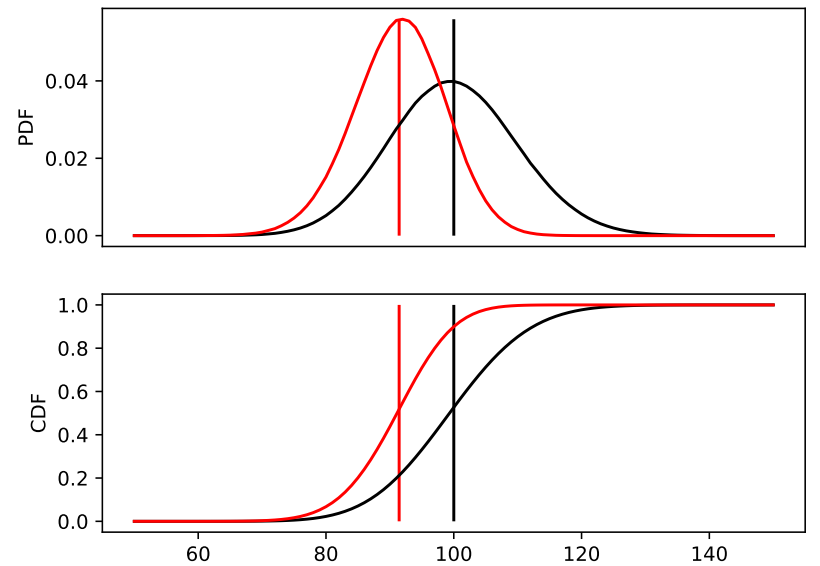

Fig. 4. Local minima's expectation bias for $\mu=100$. Black is associated with the full signal, red is associated with its local minima. Vertical lines indicate expectations of the distributions.

where

$$
C_{i}=\int_{-\infty}^{+\infty} u^{i} \varphi(u)(1-\Phi(u))^{2} d u
$$

Proof of property 3 is given in the appendix. Note that $C_{0}$ is the density of $\xi$ for a stationary signal. Numerical integration results in the following values:

$$
\left\{\begin{array}{l}
C_{0}=1 / 3 \\
C_{1} \approx-0.28209479 \\
C_{2} \approx 0.42522148
\end{array}\right.
$$

2) Occurrence of local minima: One reported on the Fig. 5 the evaluation by simulation of the probability $P_{\min }$ that a point of a linear signal, with a slope $\gamma$ and a noise variance $\sigma$, is a local minima. Notes that $P_{\min }(\gamma / \sigma=0)=C_{0}$, and $P_{\min }$ quickly decreases.

Additionally, one can derive from property 2 an upper bound on the probability that a local minima belongs to $\mathbb{F}$ :

Property 4:

$$
P(k \in \mathbb{F} \mid k \in \xi) \leq \frac{A_{k}}{P(k \in \xi)}
$$

Proof of property 4 is given in the appendix. One notices $P(k \in \xi)$ can not be too small, because in practice there are always a non negligible portion of local minima in a measurement. Moreover, Fig. 5 testifies that $\forall k \in \mathbb{F}, A_{k}$ is dramatically low. Therefore, $P(k \in \mathbb{F} \mid k \in \xi)$ is majorated by a constant close to zero, which explains a remarkable phenomenon easily noticeable through data: local minima are absent from the flanks. It is thus possible to identify points in $\overline{\mathbb{F}}$ by observing $\xi$ :

Hypothesis 2:

$$
\xi \subset \overline{\mathbb{F}}
$$




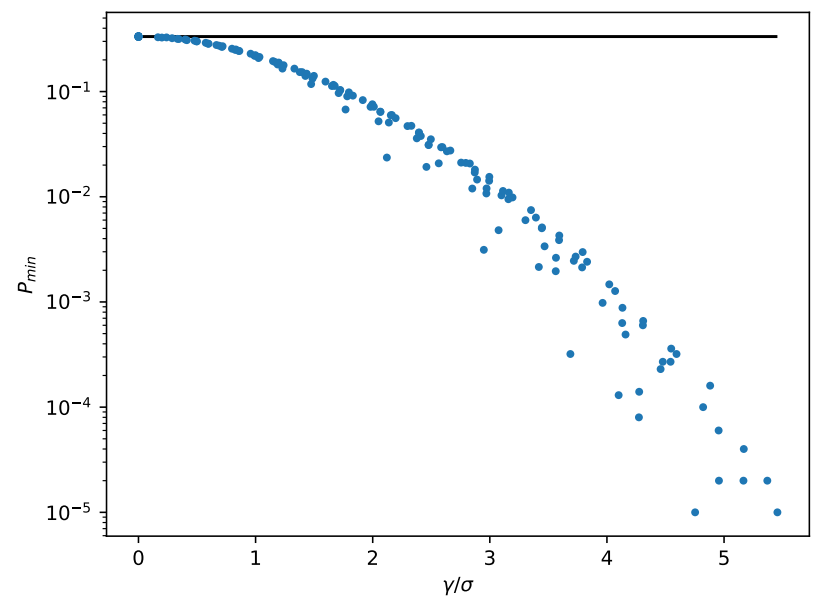

Fig. 5. Blue points represent $P_{\min }(\gamma / \sigma)$ on a grid of 20 values of $\gamma$ linearly spaced on $[0,100]$ and 20 values of $\sigma$ spaced evenly on a log scale on $[1,1000]$. Black line represents $C_{0}$.

Because it is easy to observe local minima in a given spectrum, hypothesis 2 is a convenient criterion upon which one may build an estimator for the continuum.

\section{CONTINUUM ESTIMATION}

\section{A. Intruders filtering}

In the previous section, we identified points from $\overline{\mathbb{F}}$. However, this is not exactly what we were looking for, i.e. $c$ points (where $m$ is negligible). Some undesirable intruders are present in $\xi$, as shown in Fig. 6. Indeed, it contains top points which must be removed. Moreover, local minima may accidentally appear on the flank of a significant peak. Anyway, all intruders values are substantially higher than those of the points attached to the continuum. This gives us an opportunity to filter them.

Let define the null hypothesis $H 0: \ll$ there is no discontinuity between $\xi_{i-1}$ and $\xi_{i} \gg$. Let $t_{\text {break }}$ be the $1-\alpha / 2$ order quantile of $\mathcal{N}(0,1)$ and:

$$
z_{i}=\frac{\left|\Delta y_{\xi_{i}}\right|}{\sqrt{y_{\xi_{i}}+y_{\xi_{i-1}}}}
$$

$z_{i}$ is a z-score for $H 0$, i.e. if $t_{\text {break }} \leq z_{i}$, one can reject $H 0$ with a confidence $\alpha$. By selecting a threshold $t_{\text {break }}$ for this hypothesis testing, one detects discontinuities in $\xi$, and forms groups of continuous $\xi$ sets.

Observing the sign of $\Delta y_{\xi_{i}}$ at the borders between the groups reveals groups which level is higher than those of their direct neighbours. These are intruders groups to be filtered as on Fig. 6.

\section{B. Large peaks issue}

Previous outlier filtering is able to deal with GeHP thin peaks spectra. But when faced to peaks acquired by a scintillator, peaks are very large with respect to $\mathbb{F}$ variations, and the estimator fails as shown on top plot on Fig. 7.

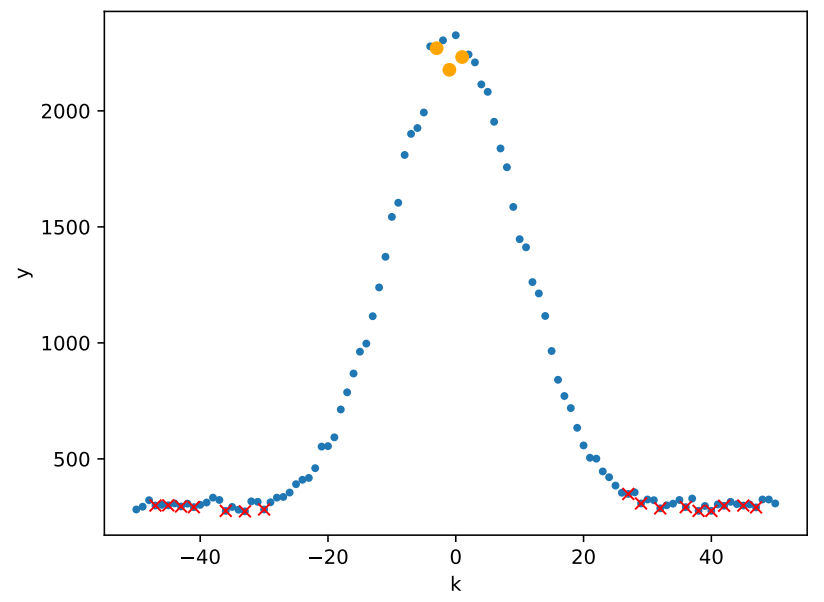

Fig. 6. Intruders filtering with $t_{\text {break }}=1.5$. Blue points represent observations $\boldsymbol{y}$, red crosses represent remaining $\xi$ after intruders filtering, orange points represent intruders.

A simple solution is to subsample the signal before the intruders filtering as shown on the middle plot on Fig. 7. It means that from the considered spectrum, one keep one point out of $p$, starting at point $s$. Parameter $p$ is the subsampling step, $s$ the subsampling offset such as $0 \leq s<p$. In this manner, the variation rate between two points is multiplied by $p$, whereas the noise level has not changed, which enables to fix large peaks issue.

To limit the information loss due to subsampling, one filters subsamples of $\xi$ successively with all possible values of $s$ for a given $p$, then merge the results as illustrated on the bottom plot of Fig. 7.

Actually, subsampling is a trick which allows to fall back on thin peaks analysis issue when faced to large peaks. An optimum choice for $p$ depends on $w$, the actual full width at half maximum (FWHM) of the peaks, and $w_{\text {opt }}$, a fix ideal FWHM that one strives to retrieve. This offers an alternative meaningful parametrization for the estimator:

$$
p=\max \left(1,\left\lfloor\frac{w}{w_{\mathrm{opt}}}\right\rfloor\right)
$$

\section{Full continuum estimation procedure}

For a given spectrum, the proposed continuum estimation procedure is the following: 1) Subsample the signal as in part III-B. 2) Observe local minima defined equation 1. 3) Filter intruders as in part III-A. 4) Correct the local minima bias by property 3. 5) Merge remaining points from each subsamples. 6) Apply a linear interpolation to extend the signal to all canals.

Parameters $t_{\text {break }}$ and $w_{\text {opt }}$ enable to define the balance between peaks and continuum. A small values for $t_{\text {break }}$ or $w_{\text {opt }}$ will tend to attribute parts of the continuum as peaks, and big values will tend to attribute peaks to the continuum. The mean width of the peaks $w$ is constant for a given 

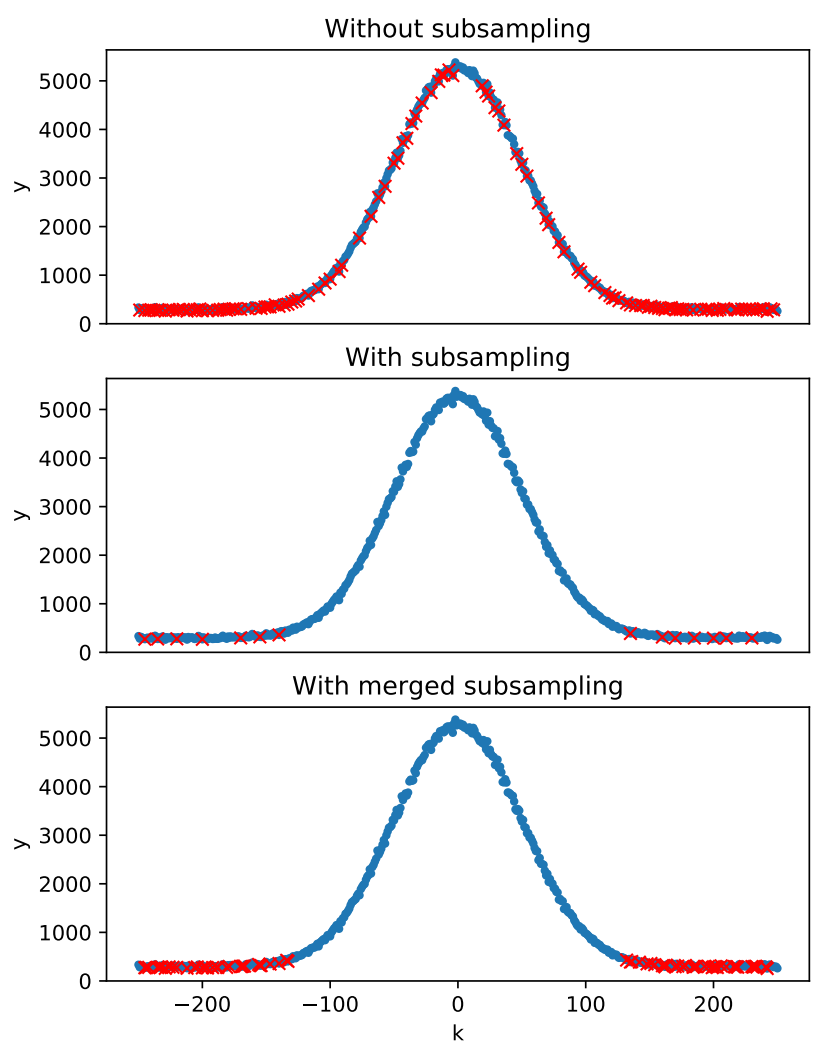

Fig. 7. Subsampling effect on outlier filtering with $t_{\text {break }}=1.5$. Top figure uses no subsamplings, middle figure uses subsampling ( $p=5, s=0)$, bottom figure uses merged subsamplings $(p=5)$. Blue line represents observations $\boldsymbol{y}$, red crosses represent remaining $\xi$ after intruders filtering.

measurement device, but still have to be specified (roughly) in order to adapt the method to the spectrometer. An extensive empirical campaign suggests to set $t_{\mathrm{break}}=1.5$ and $w_{\mathrm{opt}}=15$. The resulting full estimation is plotted on Fig. 8. One may note that the peak is essentially extracted, even if a little bit underestimated. A part of the basis of the peak is indeed attributed to the continuum which bias the estimation of the area. This is inevitably caused by the bordering areas where it is hard to attribute a point to $\mathbb{F}$ or $\overline{\mathbb{F}}$.

Ideally, one would have filtered the noise before the interpolation, and used a smooth interpolator. This would require to introduce a regularity prior. However, continuum often contains discontinuities which are difficult to take into consideration. Consequently, to find such a prior is an awkward task. For a first approach, one can simply set aside the noise and limit oneself to the padding of the signal with a linear interpolation.

\section{EXPERIMENTAL DEMONSTRATION}

Figure 1 presents real spectra continuum estimations in order to permit a comparison. Undoubtedly, the new estimator has enhanced performances and qualitatively meet our expectations

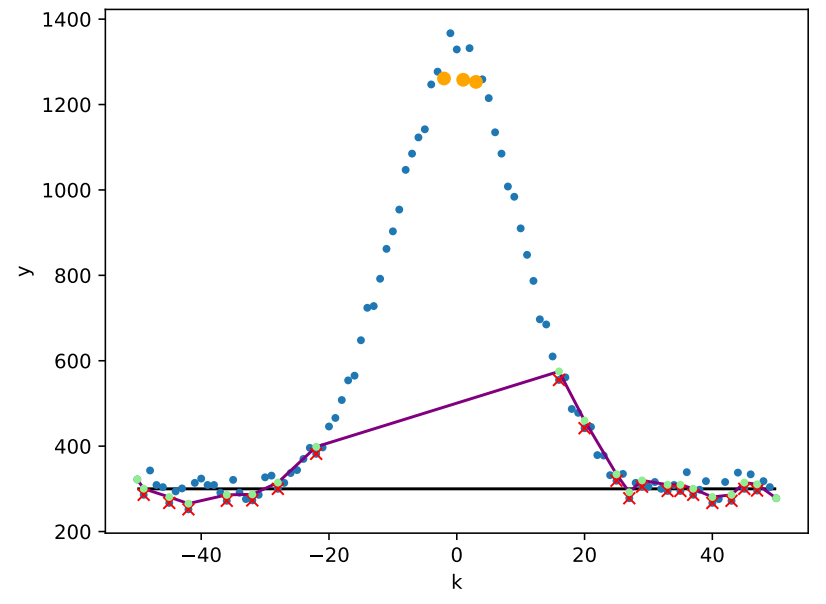

Fig. 8. Continuum estimation with $t_{\text {break }}=1.5, w_{\text {opt }}=15$ and $w=10$ Blue points represent observations $\boldsymbol{y}$, red crosses represent remaining $\xi$ after intruders filtering, orange points represent intruders, green points are the results of the bias correction, black line represents $\boldsymbol{c}$, purple line represents $\hat{\boldsymbol{c}}$.

in both cases as almost all significant peaks that one may visually identify are extracted. The method seems to conveniently handle GeHP's Compton edges and multiplets where Tervo's method failed. Furthermore, the large peaks width difference between both spectra shows the adaptability of the method. To the knowledge of the authors, there exist no relevant quantitative performance metrics for such estimators, certainly because continuum are by essence unknown quantities. One would rather extend the method to derive peaks positions and areas which are much more convenient to compare.

\section{CONCLUSION}

As a conclusion, this paper derives the properties of local minima under an original aspect, i.e. counter variations, and proposes a totally new estimation procedure, for which key parts are intruders filtering and subsampling.

This method enables a quick, adaptive and automated estimation of the continuum of a gamma spectrum. A full optimization procedure of the parameters, as well as a study of the robustness of the method would be appreciated. Nevertheless, the proposed empirical adjustment should be good enough for a general purpose, and the opinion of the authors is that little performances improvement is to be expected in this direction. Naturally, this method is showing its limitations when continuum variations are close to peaks variations.

As a conclusion, even with ideal settings, one can not ensure the goodness of the estimation, and consequently one should not use this method for quantification purpose. However, the present work stands as an improvement of the method of Tervo et al. [12]. Moreover, it would be possible to derive from this work a peaks detection method. 


\section{APPENDIX}

Proof of property 3: Let consider a stationary signal of three channels simulated with $X_{1}, X_{2}, X_{3} \sim \mathcal{N}\left(\mu, \sigma^{2}\right)$. Let $f_{X_{i}}($.$) denote their PDF and g(x)=\frac{x-\mu}{\sigma}$. Consequently:

$$
\left\{\begin{aligned}
f_{X}(x) & = & g^{\prime}(x) \varphi(g(x)) \\
P\left(X_{i} \leq x\right) & = & \Phi(g(x))
\end{aligned}\right.
$$

One is looking for $f$, the conditional PDF of $X_{2}$ when it turned out to be a local minima:

$$
\begin{aligned}
f(x) & =f_{X_{2} \mid X_{2}<X_{1}, X_{2}<X_{3}}(x) \\
& \propto f_{X_{2}}(x) P\left(x<X_{1}\right) P\left(x<X_{3}\right) \\
& \propto f_{X_{2}}(x)\left(1-P\left(X_{1}<x\right)\right)^{2} \\
& \propto g^{\prime}(x) \varphi(g(x))(1-\Phi(g(x)))^{2}
\end{aligned}
$$

Let assess the normalization constant:

$$
\int_{-\infty}^{+\infty} g^{\prime}(t) \varphi(g(t))(1-\Phi(g(t)))^{2} d t=\int_{-\infty}^{+\infty} \varphi(u)(1-\Phi(u))^{2} d u=C_{0}
$$

Thus $f(x)=\frac{1}{C_{0}} g^{\prime}(x) \varphi(g(x))(1-\Phi(g(x)))^{2}$. Let $Z$ a random variable with $f$ as PDF. Thus, involving variable mutation:

$$
\begin{aligned}
\mathbb{E}(Z)= & \int_{-\infty}^{+\infty} t f(t) d t=\int_{-\infty}^{+\infty}(\sigma g(t)+\mu) f(t) d t=\sigma \frac{C_{1}}{C_{0}}+\mu \\
\mathbb{E}\left(Z^{2}\right)= & \int_{-\infty}^{+\infty} t^{2} f(t) d t=\int_{-\infty}^{+\infty}(\sigma g(t)+\mu)^{2} f(t) d t \\
& +\infty \\
= & \int_{-\infty}\left(\sigma^{2} g(t)^{2}+2 \mu g(t)+\mu^{2}\right) f(t) d t \\
= & \sigma^{2} \frac{C_{2}}{C_{0}}+2 \mu \frac{C_{1}}{C_{0}}+\mu^{2} \\
\mathbb{V}(Z) & =\mathbb{E}\left(Z^{2}\right)-\mathbb{E}(Z)^{2} \\
& =\frac{1}{C_{0}}\left(\sigma^{2} C_{2}-\sigma C_{1}+\mu\left(2 C_{1}-C_{0}\right)+\mu^{2} C_{0}\right)
\end{aligned}
$$

Proof of property 4: One has

$$
P(k \in \mathbb{F})=P\left(k \in \mathbb{F}^{+}\right)+P\left(k \in \mathbb{F}^{-}\right)
$$

Furthermore:

$$
\begin{aligned}
P\left(k \in \xi \mid k \in \mathbb{F}^{+}\right) & =P\left(\Delta Y_{k}<0, \Delta Y_{k+1}>0 \mid \alpha \leq \Delta m_{k}\right) \\
& \leq P\left(\Delta Y_{k}<0 \mid \alpha \leq \Delta m_{k}\right)=F_{k} \leq A_{k}
\end{aligned}
$$

In the same way, one finds $P\left(k \in \xi \mid k \in \mathbb{F}^{-}\right) \leq A_{k}$.
Therefore:

$$
\begin{aligned}
P(k \in \xi \mid k \in \mathbb{F}) & =P\left(k \in \xi \mid k \in \mathbb{F}^{+}\right) \frac{P\left(k \in \mathbb{F}^{+}\right)}{P(k \in \mathbb{F})} \\
& +P\left(k \in \xi \mid k \in \mathbb{F}^{-}\right) \frac{P\left(k \in \mathbb{F}^{-}\right)}{P(k \in \mathbb{F})} \\
& \leq \frac{A_{k}}{P(k \in \mathbb{F})}\left(P\left(k \in \mathbb{F}^{+}\right)+P\left(k \in \mathbb{F}^{-}\right)\right) \\
& =A_{k}
\end{aligned}
$$

Finally:

$$
\begin{aligned}
P(k \in \mathbb{F} \mid k \in \xi) & =P(k \in \xi \mid k \in \mathbb{F}) \frac{P(k \in \mathbb{F})}{P(k \in \xi)} \\
& \leq \frac{A_{k}}{P(k \in \xi)}
\end{aligned}
$$

\section{REFERENCES}

[1] Gordon Gilmore. Practical Gamma-Ray Spectrometry. John Wiley \& Sons, 2 edition, 2011.

[2] Georg Schulze, Andrew Jirasek, Marcia ML Yu, Arnel Lim, Robin FB Turner, and Michael W Blades. Investigation of selected baseline removal techniques as candidates for automated implementation, volume 59. SAGE Publications Sage UK: London, England, 2005.

[3] MA Mariscotti. A method for automatic identification of peaks in the presence of background and its application to spectrum analysis, volume 50. Elsevier, 1967.

[4] I De Lotto and A Ghirardi. Automatic peak-locating techniques for $\gamma$-ray spectra, volume 143. Elsevier, 1977.

[5] A Robertson, WV Prestwich, and TJ Kennett. An automatic peakextraction technique, volume 100. Elsevier, 1972.

[6] WW Black. Application of correlation techniques to isolate structures in experimental data, volume 71. Elsevier, 1969.

[7] AL Connelly and WW Black. Automatic location and area determination of photopeaks, volume 82. Elsevier, 1970.

[8] F Rossitto and M Terrani. Convolution analysis of pulse-height distributions, volume 79. Elsevier, 1970.

[9] $\mathrm{T}$ Inouye, $\mathrm{T}$ Harper, and NC Rasmussen. Application of fourier transforms to the analysis of spectral data. Nuclear Instruments and Methods, 67(1):125-132, 1969.

[10] W Westmeier. Background subtraction in ge (li) gamma-ray spectra. Nuclear Instruments and Methods, 180(1):205-210, 1981.

[11] TJ Kennett, WV Prestwich, and RJ Tervo. Automated analysis for high energy gamma ray spectra. Nuclear Instruments and Methods in Physics Research, 190(2):313-323, 1981.

[12] RJ Tervo, TJ Kennett, and WV Prestwich. An automated background estimation procedure for gamma ray spectra. Nuclear Instruments and Methods in Physics Research, 216(1-2):205-218, 1983.

[13] LV East, RL Phillips, and AR Strong. A fresh approach to NaI scintillation detector spectrum analysis, volume 193. Elsevier, 1982.

[14] SA Gerasimov. Recursive filtering of gamma ray spectra, volume 72. Elsevier, 1992.

[15] Miroslav Morháč. An algorithm for determination of peak regions and baseline elimination in spectroscopic data. Nuclear Instruments and Methods in Physics Research Section A: Accelerators, Spectrometers, Detectors and Associated Equipment, 600(2):478-487, 2009.

[16] Zhi-Min Zhang, Shan Chen, and Yi-Zeng Liang. Baseline correction using adaptive iteratively reweighted penalized least squares. Analyst, 135(5):1138-1146, 2010.

[17] Eric Barat, Thomas Dautremer, and Thierry Montagu. Nonparametric bayesian inference in nuclear spectrometry. In 2007 IEEE Nuclear Science Symposium Conference Record, volume 1, pages 880-887. IEEE, 2007.

[18] Canberra. Genie2000 v3.1. Customization tools manual, 2006.

[19] ISO-19017:2015(en). Guidance for gamma spectrometry measurement of radioactive waste, 2015.

[20] Gilbert Saporta. Probabilités, analyse des données et statistique. Editions Technip, 2006 